\title{
Evolutionary models for cool brown dwarfs and extrasolar giant planets. The case of HD 209458
}

\author{
I. Baraffe ${ }^{1,2}$, G. Chabrier ${ }^{1}$, T. S. Barman ${ }^{3}$, F. Allard ${ }^{1}$, and P. H. Hauschildt ${ }^{4}$ \\ 1 C.R.A.L (UMR 5574 CNRS), École Normale Supérieure, 69364 Lyon Cedex 07, France \\ e-mail: ibaraffe, chabrier, fallard@ens-lyon.fr \\ 2 Max-Planck-Institut für Astrophysik, Karl-Schwarzschildstr.1, 85748 Garching, Germany \\ 3 Department of Physics, Wichita State University, Wichita, KS 67260-0032, USA \\ e-mail: travis.barman@wichita.edu \\ ${ }^{4}$ Hamburger Sternwarte, Gojenbergsweg 112, 21029 Hamburg, Germany \\ e-mail: phauschildt@hs.uni-hamburg.de
}

Received 27 November 2002 / Accepted 18 February 2003

\begin{abstract}
We present evolutionary models for cool brown dwarfs and extra-solar giant planets. The models reproduce the main trends of observed methane dwarfs in near-IR color-magnitude diagrams. We also present evolutionary models for irradiated planets, coupling for the first time irradiated atmosphere profiles and inner structures. We focus on HD 209458-like systems and show that irradiation effects can substantially affect the radius of sub-jovian mass giant planets. Irradiation effects, however, cannot alone explain the large observed radius of HD 209458b. Adopting assumptions which optimise irradiation effects and taking into account the extension of the outer atmospheric layers, we still find $\sim 20 \%$ discrepancy between observed and theoretical radii. An extra source of energy seems to be required to explain the observed value of the first transit planet.
\end{abstract}

Key words. planetary systems - stars: brown dwarfs - stars: evolution - stars: individual (HD 209458)

\section{Introduction}

The past decade was marked by two major discoveries in the field of stellar and planetary physics: the detections of the first unambiguous brown dwarf (BD) GL 229B (Oppenheimer et al. $1995)$ and the first extrasolar giant planet (EGP) 51 Peg b (Mayor \& Queloz 1995). The near-IR spectrum of GL 229B was found to be dominated by strong methane absorption bands, looking more similar to Jupiter than to late type-stars. On the other hand, the surprisingly small orbital separation between $51 \mathrm{Peg} \mathrm{b}$ and its parent star suggests that the planet should be affected by irradiation and that, given the expected large surface temperature, its atmospheric properties should resemble more the ones of relatively hot brown dwarfs than the ones of jovian planets.

Since then, about thirty methane dwarfs (or the so-called T-dwarfs) have been identified, due mainly to the near-IR surveys 2MASS (Burgasser et al. 1999), SDSS (Strauss et al. 1999) and the VLT (Cuby et al. 1999). The radial velocity technique has now revealed more than 100 EGPs in orbit around nearby stars (see Hubbard et al. 2002 for a review and references therein), with a large fraction $(\sim 10 \%-20 \%)$ being extremely close (less than $0.06 \mathrm{AU}$ ) to their parent star. The mass of substellar companions detected by radial velocimetry extends well above the deuterium burning minimum mass

Send offprint requests to: I. Baraffe, e-mail: ibaraffe@ens-lyon.fr
$0.012 M_{\odot}=12 M_{\mathrm{J}}$ (Saumon et al. 1996; Chabrier et al. 2000a). This mass is often used as the boundary between planets and brown dwarfs, more for semantical than physical reasons. On the other hand isolated objects with planetary masses are now discovered in young stellar clusters, down to a few $M_{\mathrm{J}}$, as recently reported by Zapatero et al. (2002) in $\sigma$ Orionis. These observations suggest that there is an overlap between the mass range of the least massive brown dwarfs and of the most massive giant planets. In principle, different formation processes should distinguish planets from brown dwarfs. However, such a distinction is difficult to characterize in terms of atmospheric, structural and cooling properties since both types of objects have convective interiors with essentially a metallic $\mathrm{H} / \mathrm{He}$ mixture. The signature of a central rock+ice core, like in solar giant planets, would be the clear identification of a planet. The presence of a core can affect the radius of a planet, yielding a smaller planetary radius than in the absence of a core. For $1 M_{\mathrm{J}}$, the effect is about $5 \%$ on the radius for a core mass $<0.06 M_{\mathrm{J}}$ (see Saumon et al. 1996). The presence of this core can be inferred from the accurate characterization of the gravitational moments of the object, and such an observation is currently not feasible for EGPs. In addition, both giant planets and brown dwarfs have atmospheres dominated by molecular absorption and the effects of cloud formation. Although frustrating from an observational point of view, these similarities imply that the general cooling theory for BDs, involving detailed models of the atmosphere and inner structures, can be applied to EGPs. 
In terms of cooling properties, this general theory can even be applied to Jupiter, as emphasized by Hubbard et al. (2002). Additional observational constraints, as provided by spacecraft encounters or by direct probes (e.g. Galileo), have lead to refinements of the models (heavy element core, non-standard chemical composition). As mentioned above, such constraints are, unfortunately, far from being accessible for EGPs.

Much effort has been devoted to the modeling of substellar objects during the past decade, improving our understanding of cool atmospheres (see Allard et al. 1997 for a review), of the role of dust (Tsuji et al. 1999; Burrows et al. 2000; Ackerman \& Marley 2001; Allard et al. 2001; Marley et al. 2002), of irradiation (Saumon et al. 1996; Seager \& Sasselov 1998; Sudarsky et al. 2000; Barman et al. 2001), and of their inner structure and evolutionary properties (Burrows et al. 1997; Chabrier et al. 2000b; see Chabrier \& Baraffe 2000 for a review). One remaining major challenge in the theory is the description of dynamical processes of grain formation and diffusion necessary to understand the transition between L-dwarfs and T-dwarfs, which is expected to take place at $T_{\text {eff }} \sim 1300 \mathrm{~K}-1700 \mathrm{~K}$. The former objects are better reproduced by dusty atmosphere models, whereas the later are better reproduced by dust-free (or partly dusty) models. The recent observations of $\mathrm{L} / \mathrm{T}$ dwarfs at the transition clearly indicate that complex processes take place in the atmosphere of these objects (see e.g. Burgasser et al. 2002). Another important challenge is the modeling of irradiation effects, which are expected to affect the spectra of close-in EGPs, and may also affect their inner structure and cooling properties. The recent discovery of the planet HD 209458b transiting its parent star (Charbonneau et al. 2000) provides a unique test to explore such effects, since its mass and radius can be determined with high accuracy from the modeling of the transit lightcurve. According to the most recent determination (Cody $\&$ Sasselov 2002), the mass and radius of the planet are estimated to be $m=0.69 \pm 0.02 M_{\mathrm{J}}$ and $R=1.42_{-0.13}^{+0.10} R_{\mathrm{J}}$.

Evolutionary models including crude estimates of the effects of irradiation on planet atmospheres suggest that extrinsic heating is sufficient to maintain a larger planetary radius compared to an isolated planet. It has thus been argued that irradiation could explain the large radius of HD 209458b (Guillot et al. 1996; Burrows et al. 2000). More recently, Guillot \& Showman (2002) questioned such results and argue that the radius of HD $209458 \mathrm{~b}$ can only be reproduced if the deep atmosphere is much hotter than what can be expected from irradiation effects. However, none of these calculations includes a consistent treatment between the irradiated atmospheric structure and the interior structure of the planet. Such a consistent treatment is mandatory to get reliable results since the deep interior entropy profile, which determines the heat content of the planet to be radiated away while it cools, is affected by the modification of the atmospheric temperature profile due to the incoming external heat flux. The main goal of the present paper is to present the first such consistent calculations. As mentioned above, in the case of non-irradiation, these calculations apply to the evolution of cool (dust-free like) brown dwarfs, i.e. T-dwarfs, and extrasolar giant planets far enough from the parent star for the irradiation effects on the thermal structure of the planet to be negligible. This is the case of the solar giant planets, the cooling of which is simply characterized by the cooling properties of the "isolated" planet plus the heating contribution from the Sun $4 \pi \sigma R_{\mathrm{p}}^{2} T_{\odot}^{4}$, where $R_{\mathrm{p}}$ is the radius of the planet and $T_{\odot}$ represents the equivalent black body temperature of the converted solar radiation (Hubbard 1977; Guillot et al. 1995). In Sect. 2 we briefly present the input physics of non-irradiated models, describing methane dwarfs and isolated EGPs. Apart from the impinging stellar flux, the same input physics are used to analyse the effects of irradiation. The effects on the radius and cooling properties of giant planets are described in Sect. 3 and results are compared to the observed properties of HD 209458b. Discussion follows in Sect. 4.

\section{Non-irradiated models}

\subsection{Model description}

The main input physics involved in the present models are the same as described in our previous works (Chabrier \& Baraffe 1997; Baraffe et al. 1998; Chabrier et al. 2000b). The models are based on the coupling between interior and non-grey atmosphere structures. The treatment of dust in the atmosphere is described in detail in Allard et al. (2001), with two limiting cases of dust treatment. The first case, referred to as "DUSTY", takes into account the formation of dust in the equation of state, and its scattering and absorption in the radiative transfer equation. Such models assume that dust species remain where they form, according to the chemical equilibrium conditions. The second case, referred to as "COND", neglects dust opacity in the radiative transfer equation. In a previous paper (Chabrier et al. 2000b), we presented the evolutionary models based on DUSTY atmosphere models, aimed at describing the evolution and the photometric and spectroscopic properties of early L-dwarfs. The present paper is devoted to evolutionary models based on the COND approach, which are more appropriate to objects with effective temperatures $T_{\text {eff }} \lesssim 1300 \mathrm{~K}$, such as methane dwarfs or EGPs at large orbital separation. These models apply when all grains have gravitationally settled below the photosphere. A forthcoming paper will be devoted to models taking into account characteristic diffusion timescales of different processes affecting the dust stratification (e.g. coagulation, gravitational settling, convection). These models aim at describing in particular the transition objects between late L-dwarfs and early T-dwarfs (see Allard 2002).

\subsection{Evolution of cool brown dwarfs}

A preliminary version of the COND models was presented in Chabrier et al. (2000b) down to $0.01 M_{\odot}$. In the present paper, we extend our calculations to $T_{\text {eff }}=100 \mathrm{~K}$ and $m=0.5 M_{\mathrm{J}}$. The evolution of $L$ and $T_{\text {eff }}$ as a function of time for different masses is displayed in Fig. 1. The properties of the COND models for different ages are given in Tables 1-5. As already stressed in Chabrier et al. (2000b), the treatment of dust in the atmospheric models barely affects the evolutionary tracks in terms of $L$ and $T_{\text {eff }}$ as a function of time for a given mass (see Fig. 2 of Chabrier et al. 2000b). Consequently, although the COND models are more appropriate to describe the spectral 


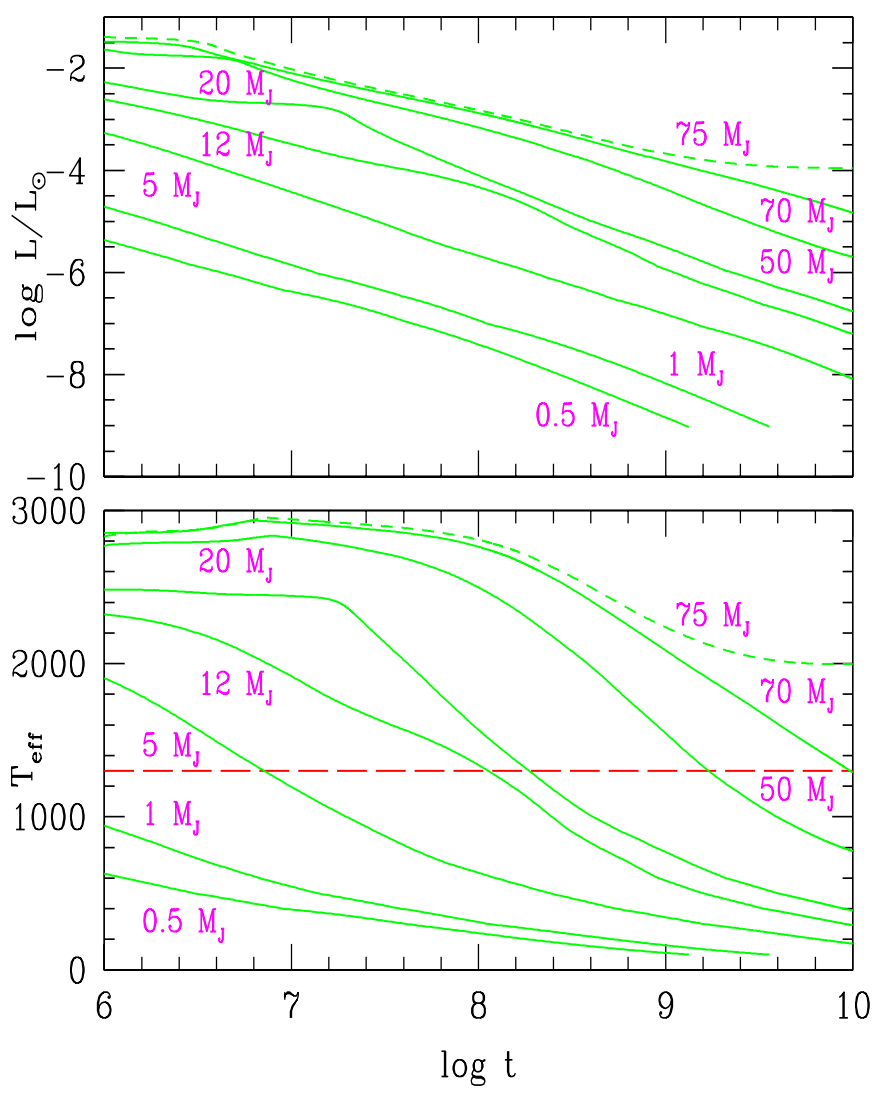

Fig. 1. Evolution of the luminosity $L$ and effective temperature $T_{\text {eff }}$ as a function of time (in yr) for different masses. Substellar objects are indicated by solid lines and stars by short-dashed lines. The horizontal long-dashed line indicates the limit $\left(T_{\text {eff }} \sim 1300 \mathrm{~K}\right)$ below which the COND models are appropriate for the photometric and spectroscopic description of T-dwarfs and EGPs (see text).

and photometrical properties of substellar objects with $T_{\text {eff }} \lesssim$ $1300 \mathrm{~K}$, they provide a good description of their cooling properties even at higher $T_{\text {eff }}$. In other words, it is not necessary to perform evolutionary calculations with the DUSTY models above $T_{\text {eff }} \sim 1300 \mathrm{~K}$ and switch to the COND models below. An object characteristic of the present models was recently discovered by Zapatero et al. (2002) in $\sigma$ Orionis: S Ori 70. From a comparison of its observed spectrum with COND synthetic spectra (Allard et al. 2001), Zapatero et al. (2002) estimate an effective temperature $T_{\text {eff }} \sim 700-1000 \mathrm{~K}$. If the membership of S Ori 70 to $\sigma$ Orionis is confirmed, implying an age $<10 \mathrm{Myr}$, its mass mass should be $\$ 5 M_{\mathrm{J}}$ (see Fig. 1 ).

Several methane dwarfs have been discovered in the solar neighbourhood, implying older ages and thus larger masses than the extreme case of S Ori 70. At an age of $10^{8} \mathrm{yr}$, only objects with masses below the deuterium burning minimum mass $\left(m \leq 0.012 M_{\odot}\right)$ have $T_{\text {eff }} \lessgtr 1300 \mathrm{~K}$, whereas at $5 \mathrm{Gyr}$, it is the case for all substellar objects with $m \lesssim 0.06 M_{\odot}$ (see Fig. 1 and Tables 1 and 4). Photometric observations and parallax determinations of several L- and T-dwarfs (Els et al. 2001; Leggett et al. 2002a; Dahn et al. 2002) now allow a comparison with models in observational color-magnitude diagrams (CMD), providing stringent constraints on theoretical models (see Figs. 2-4).

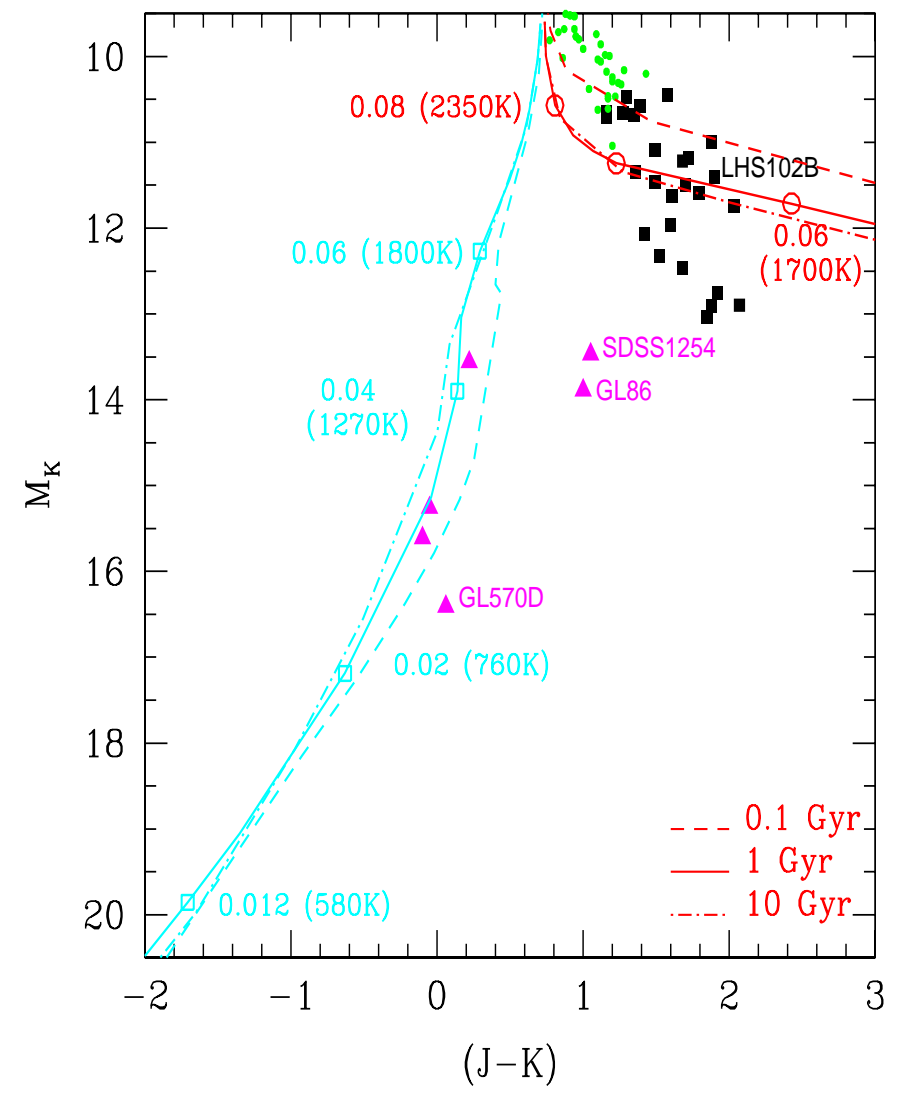

Fig. 2. Color - Magnitude diagram $(J-K)-M_{\mathrm{K}}$. Observations are taken from Leggett (1992) (mostly for M-dwarfs) and Dahn et al. (2002). Also shown: LHS 102B (Goldman et al. 1999), GL86 (Els et al. 2001). M-dwarfs are shown by dots, L-dwarfs by filled squares and T-dwarfs by triangles. DUSTY isochrones (Chabrier et al. 2000b) are displayed in the upper right part of the figure, for different ages, as indicated. The COND isochrones are displayed in the left part of the figure. Some masses (in $M_{\odot}$ ) and their corresponding $T_{\text {eff }}$ are indicated on the $1 \mathrm{Gyr}$ isochrones by open squares (COND) and open circles (DUSTY). The names of two L/T transition objects and of the faintest T-dwarf known with parallax are indicated.

As already noticed in Allard et al. (1996) for GL 229B, models free of atmospheric dust clouds better reproduce the near-IR photometric and spectral properties of methane dwarfs. This is illustrated in $(J-K)$ and $\left(K-L^{\prime}\right)$ colors in Figs. 2 and 3 respectively, where the COND models reproduce the main observed trends. In Fig. 2, we note the two transition objects, intermediate between L- and T- dwarfs, with $(J-K) \sim 1$ (GL 86B: Els et al. 1999; SDSS 1254-01: Leggett et al. 2002a, Dahn et al. 2002) and the faintest L-dwarfs (Dahn et al. 2002), which are not described by either the DUSTY or COND limiting cases, and require a detailed treatment of dust diffusion in the atmosphere, as mentioned in Sect. 2.1.

The predictions of the COND models provide a general good agreement with observed near-IR photometry and spectra at wavelength $>1 \mu \mathrm{m}$ (Leggett et al. 2002b; Zapatero et al. 2002). The models show however shortcomings at shorter 
Table 1. COND isochrones for $0.1 \mathrm{Gyr}$.

\begin{tabular}{|c|c|c|c|c|c|c|c|c|c|c|c|c|}
\hline$m / M_{\odot}$ & $T_{\text {eff }}$ & $\log L / L_{\odot}$ & $R / R_{\odot}$ & $\log g$ & $M_{V}$ & $M_{R}$ & $M_{I}$ & $M_{J}$ & $M_{H}$ & $M_{K}$ & $M_{L^{\prime}}$ & $M_{M}$ \\
\hline 0.0005 & 240. & -7.418 & 0.114 & 3.020 & 41.98 & 37.51 & 34.00 & 28.42 & 26.59 & 37.66 & 19.57 & 17.64 \\
\hline 0.0010 & 309. & -6.957 & 0.117 & 3.300 & 32.58 & 28.68 & 25.89 & 22.43 & 22.38 & 29.11 & 17.41 & 15.69 \\
\hline 0.0020 & 425. & -6.383 & 0.120 & 3.580 & 29.69 & 25.62 & 22.79 & 20.05 & 19.76 & 23.13 & 15.94 & 14.55 \\
\hline 0.0030 & 493. & -6.112 & 0.121 & 3.746 & 28.71 & 24.48 & 21.66 & 18.88 & 18.57 & 20.88 & 15.21 & 13.93 \\
\hline 0.0040 & 563. & -5.880 & 0.122 & 3.869 & 28.09 & 23.77 & 20.95 & 17.95 & 17.71 & 19.35 & 14.59 & 13.50 \\
\hline 0.0050 & 630. & -5.686 & 0.122 & 3.965 & 27.65 & 23.25 & 20.44 & 17.23 & 17.02 & 18.15 & 14.06 & 13.14 \\
\hline 0.0060 & 688. & -5.534 & 0.121 & 4.048 & 27.36 & 22.92 & 20.09 & 16.71 & 16.51 & 17.26 & 13.67 & 12.83 \\
\hline 0.0070 & 760. & -5.365 & 0.121 & 4.117 & 27.03 & 22.55 & 19.74 & 16.16 & 16.01 & 16.38 & 13.26 & 12.55 \\
\hline 0.0080 & 816. & -5.246 & 0.120 & 4.180 & 26.77 & 22.28 & 19.49 & 15.76 & 15.65 & 15.79 & 12.97 & 12.35 \\
\hline 0.0090 & 886. & -5.103 & 0.120 & 4.232 & 26.45 & 21.96 & 19.19 & 15.32 & 15.23 & 15.16 & 12.63 & 12.13 \\
\hline 0.0100 & 953. & -4.978 & 0.120 & 4.279 & 26.10 & 21.66 & 18.92 & 14.94 & 14.86 & 14.69 & 12.34 & 11.96 \\
\hline 0.0120 & 1335. & -4.332 & 0.129 & 4.297 & 23.53 & 19.44 & 16.79 & 13.20 & 12.97 & 12.76 & 10.90 & 11.17 \\
\hline 0.0150 & 1399. & -4.281 & 0.124 & 4.424 & 23.30 & 19.24 & 16.46 & 13.05 & 12.82 & 12.65 & 10.83 & 11.15 \\
\hline 0.0200 & 1561. & -4.110 & 0.122 & 4.569 & 22.30 & 18.55 & 16.08 & 12.60 & 12.34 & 12.17 & 10.53 & 10.99 \\
\hline 0.0300 & 1979. & -3.668 & 0.126 & 4.715 & 19.96 & 16.80 & 14.48 & 11.52 & 11.20 & 10.90 & 9.82 & 10.38 \\
\hline 0.0400 & 2270. & -3.386 & 0.132 & 4.797 & 18.46 & 15.63 & 13.31 & 10.89 & 10.52 & 10.19 & 9.39 & 9.84 \\
\hline 0.0500 & 2493. & -3.167 & 0.141 & 4.837 & 17.09 & 14.77 & 12.53 & 10.43 & 10.02 & 9.71 & 9.04 & 9.37 \\
\hline 0.0600 & 2648. & -3.008 & 0.150 & 4.863 & 16.08 & 14.12 & 12.01 & 10.10 & 9.68 & 9.37 & 8.78 & 9.03 \\
\hline 0.0700 & 2762. & -2.879 & 0.160 & 4.874 & 15.33 & 13.59 & 11.60 & 9.82 & 9.39 & 9.10 & 8.55 & 8.75 \\
\hline 0.0720 & 2782. & -2.856 & 0.162 & 4.875 & 15.20 & 13.50 & 11.53 & 9.77 & 9.34 & 9.05 & 8.51 & 8.70 \\
\hline 0.0750 & 2809. & -2.821 & 0.166 & 4.875 & 15.01 & 13.36 & 11.42 & 9.69 & 9.26 & 8.97 & 8.44 & 8.63 \\
\hline 0.0800 & 2846. & -2.776 & 0.170 & 4.880 & 14.77 & 13.18 & 11.29 & 9.60 & 9.16 & 8.87 & 8.36 & 8.53 \\
\hline 0.0900 & 2910. & -2.689 & 0.180 & 4.884 & 14.34 & 12.85 & 11.03 & 9.40 & 8.96 & 8.68 & 8.19 & 8.35 \\
\hline 0.1000 & 2960. & -2.617 & 0.189 & 4.887 & 14.02 & 12.58 & 10.82 & 9.24 & 8.80 & 8.52 & 8.05 & 8.19 \\
\hline
\end{tabular}

Notes: $T_{\text {eff }}$ is in K, the gravity $g$ in cgs. The VRI magnitudes are in the Johnson-Cousins system (Bessell 1990), JHK in the CIT system (Leggett 1992), $L^{\prime}$ in the Johnson-Glass system and $M$ in the Johnson system.

Table 2. Same as Table 1 for 0.5 Gyr.

\begin{tabular}{|c|c|c|c|c|c|c|c|c|c|c|c|c|}
\hline$m / M_{\odot}$ & $T_{\text {eff }}$ & $\log L / L_{\odot}$ & $R / R_{\odot}$ & $\log g$ & $M_{V}$ & $M_{R}$ & $M_{I}$ & $M_{J}$ & $M_{H}$ & $M_{K}$ & $M_{L^{\prime}}$ & $M_{M}$ \\
\hline 0.0005 & 141. & -8.415 & 0.105 & 3.097 & 56.30 & 51.03 & 46.60 & 37.42 & 33.07 & 51.62 & 23.09 & 20.59 \\
\hline 0.0010 & 203. & -7.753 & 0.109 & 3.365 & 47.57 & 42.88 & 38.99 & 31.61 & 29.15 & 43.23 & 20.93 & 18.68 \\
\hline 0.0020 & 272. & -7.218 & 0.112 & 3.639 & 37.05 & 33.00 & 30.06 & 25.07 & 24.62 & 34.02 & 18.66 & 16.58 \\
\hline 0.0030 & 322. & -6.913 & 0.113 & 3.805 & 32.02 & 28.23 & 25.75 & 22.05 & 22.27 & 29.03 & 17.52 & 15.53 \\
\hline 0.0040 & 370. & -6.670 & 0.114 & 3.928 & 30.65 & 26.73 & 24.16 & 21.01 & 21.06 & 26.45 & 16.85 & 15.05 \\
\hline 0.0050 & 409. & -6.496 & 0.113 & 4.027 & 29.60 & 25.57 & 22.94 & 20.20 & 20.11 & 24.54 & 16.32 & 14.67 \\
\hline 0.0060 & 449. & -6.340 & 0.113 & 4.112 & 29.16 & 25.05 & 22.39 & 19.64 & 19.51 & 23.10 & 15.92 & 14.36 \\
\hline 0.0070 & 488. & -6.200 & 0.112 & 4.185 & 28.71 & 24.51 & 21.80 & 19.10 & 18.91 & 21.68 & 15.52 & 14.06 \\
\hline 0.0080 & 525. & -6.080 & 0.111 & 4.249 & 28.40 & 24.14 & 21.41 & 18.65 & 18.46 & 20.74 & 15.19 & 13.83 \\
\hline 0.0090 & 564. & -5.963 & 0.110 & 4.307 & 28.14 & 23.82 & 21.07 & 18.21 & 18.04 & 19.95 & 14.88 & 13.63 \\
\hline 0.0100 & 599. & -5.864 & 0.110 & 4.358 & 27.91 & 23.53 & 20.77 & 17.80 & 17.66 & 19.22 & 14.59 & 13.43 \\
\hline 0.0120 & 759. & -5.447 & 0.110 & 4.432 & 27.20 & 22.73 & 19.91 & 16.38 & 16.29 & 16.82 & 13.51 & 12.68 \\
\hline 0.0150 & 791. & -5.404 & 0.107 & 4.557 & 27.11 & 22.63 & 19.81 & 16.20 & 16.16 & 16.58 & 13.41 & 12.60 \\
\hline 0.0200 & 936. & -5.133 & 0.104 & 4.704 & 26.53 & 22.07 & 19.27 & 15.33 & 15.34 & 15.37 & 12.78 & 12.21 \\
\hline 0.0300 & 1264. & -4.636 & 0.101 & 4.905 & 24.97 & 20.77 & 17.95 & 13.90 & 13.87 & 13.69 & 11.70 & 11.68 \\
\hline 0.0400 & 1583. & -4.255 & 0.100 & 5.040 & 23.11 & 19.28 & 16.74 & 12.92 & 12.76 & 12.68 & 10.95 & 11.33 \\
\hline 0.0500 & 1875. & -3.955 & 0.101 & 5.131 & 21.31 & 17.94 & 15.53 & 12.21 & 11.95 & 11.79 & 10.44 & 10.99 \\
\hline 0.0600 & 2116. & -3.729 & 0.102 & 5.194 & 19.99 & 16.91 & 14.50 & 11.69 & 11.36 & 11.13 & 10.10 & 10.63 \\
\hline 0.0700 & 2329. & -3.534 & 0.106 & 5.233 & 18.84 & 16.04 & 13.68 & 11.27 & 10.90 & 10.63 & 9.81 & 10.26 \\
\hline 0.0720 & 2369. & -3.498 & 0.107 & 5.238 & 18.60 & 15.89 & 13.54 & 11.19 & 10.81 & 10.54 & 9.75 & 10.19 \\
\hline 0.0750 & 2426. & -3.445 & 0.108 & 5.244 & 18.25 & 15.66 & 13.33 & 11.08 & 10.69 & 10.42 & 9.67 & 10.08 \\
\hline 0.0800 & 2518. & -3.356 & 0.111 & 5.248 & 17.65 & 15.27 & 13.00 & 10.89 & 10.49 & 10.22 & 9.53 & 9.89 \\
\hline 0.0900 & 2680. & -3.189 & 0.119 & 5.241 & 16.54 & 14.56 & 12.43 & 10.55 & 10.12 & 9.85 & 9.25 & 9.53 \\
\hline 0.1000 & 2804. & -3.047 & 0.128 & 5.223 & 15.68 & 13.96 & 11.98 & 10.25 & 9.80 & 9.54 & 9.00 & 9.22 \\
\hline
\end{tabular}

wavelength, with a flux excess around $0.8-0.9 \mu \mathrm{m}$, character- significantly bluer $(I-J)$ colors than observations. As menistic of the $I$-bandpass. This problem is illustrated in Fig. 4 tioned in Allard et al. (2001), uncertainties in the current in a $(I-J)-M_{\mathrm{J}} \mathrm{CMD}$, where the COND models predict treatment of the far wings of the absorption lines of alkali 
Table 3. Same as Table 1 for 1 Gyr.

\begin{tabular}{|c|c|c|c|c|c|c|c|c|c|c|c|c|}
\hline$m / M_{\odot}$ & $T_{\text {eff }}$ & $\log L / L_{\odot}$ & $R / R_{\odot}$ & $\log g$ & $M_{V}$ & $M_{R}$ & $M_{I}$ & $M_{J}$ & $M_{H}$ & $M_{K}$ & $M_{L^{\prime}}$ & $M_{M}$ \\
\hline 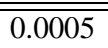 & 1111. & $=-8.851$ & 0.102 & 3.115 & 60.75 & 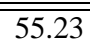 & 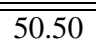 & 40.19 & 35.07 & "55.87 & 24.15 & $\overline{21.49}$ \\
\hline 0.0010 & 160. & -8.185 & 0.106 & 3.386 & 54.15 & 49.10 & 44.69 & 35.58 & 32.06 & 49.17 & 22.40 & 19.95 \\
\hline 0.0020 & 226. & -7.560 & 0.109 & 3.662 & 44.39 & 39.91 & 36.34 & 29.28 & 27.80 & 40.31 & 20.18 & 17.94 \\
\hline 0.0030 & 270. & -7.244 & 0.111 & 3.827 & 37.64 & 33.60 & 30.73 & 25.29 & 24.99 & 34.51 & 18.84 & 16.66 \\
\hline 0.0040 & 304. & -7.031 & 0.111 & 3.950 & 32.62 & 28.93 & 26.60 & 22.49 & 22.91 & 30.25 & 17.91 & 15.73 \\
\hline 0.0050 & 342. & -6.831 & 0.110 & 4.051 & 31.58 & 27.79 & 25.36 & 21.71 & 21.98 & 28.28 & 17.38 & 15.36 \\
\hline 0.0060 & 377. & -6.664 & 0.110 & 4.134 & 30.53 & 26.63 & 24.12 & 20.96 & 21.07 & 26.39 & 16.87 & 15.01 \\
\hline 0.0070 & 403. & -6.556 & 0.109 & 4.208 & 29.77 & 25.79 & 23.26 & 20.41 & 20.43 & 25.04 & 16.54 & 14.76 \\
\hline 0.0080 & 438. & -6.417 & 0.108 & 4.272 & 29.37 & 25.31 & 22.73 & 19.93 & 19.89 & 23.77 & 16.18 & 14.50 \\
\hline 0.0090 & 464. & -6.325 & 0.107 & 4.331 & 29.06 & 24.94 & 22.33 & 19.59 & 19.49 & 22.85 & 15.93 & 14.31 \\
\hline 0.0100 & 491. & -6.235 & 0.107 & 4.383 & 28.74 & 24.55 & 21.89 & 19.23 & 19.07 & 21.90 & 15.65 & 14.11 \\
\hline 0.0120 & 578. & -5.955 & 0.106 & 4.467 & 28.09 & 23.75 & 21.01 & 18.15 & 18.03 & 19.86 & 14.88 & 13.60 \\
\hline 0.0150 & 628. & -5.835 & 0.103 & 4.587 & 27.86 & 23.47 & 20.72 & 17.70 & 17.62 & 19.05 & 14.56 & 13.38 \\
\hline 0.0200 & 766. & -5.514 & 0.100 & 4.736 & 27.31 & 22.85 & 20.05 & 16.56 & 16.55 & 17.19 & 13.72 & 12.80 \\
\hline 0.0300 & 1009. & -5.071 & 0.096 & 4.948 & 26.40 & 21.96 & 19.15 & 15.10 & 15.16 & 15.14 & 12.67 & 12.15 \\
\hline 0.0400 & 1271. & -4.696 & 0.093 & 5.099 & 25.19 & 20.99 & 18.13 & 14.04 & 14.04 & 13.90 & 11.88 & 11.80 \\
\hline 0.0500 & 1543. & -4.374 & 0.092 & 5.211 & 23.73 & 19.81 & 17.15 & 13.21 & 13.12 & 13.04 & 11.25 & 11.53 \\
\hline 0.0600 & 1801. & -4.106 & 0.092 & 5.291 & 22.13 & 18.59 & 16.10 & 12.56 & 12.36 & 12.27 & 10.77 & 11.26 \\
\hline 0.0700 & 2082. & -3.829 & 0.094 & 5.333 & 20.44 & 17.31 & 14.87 & 11.93 & 11.62 & 11.43 & 10.32 & 10.85 \\
\hline 0.0720 & 2140. & -3.772 & 0.095 & 5.336 & 20.12 & 17.05 & 14.61 & 11.80 & 11.48 & 11.27 & 10.23 & 10.75 \\
\hline 0.0750 & 2234. & -3.679 & 0.098 & 5.334 & 19.59 & 16.63 & 14.21 & 11.60 & 11.25 & 11.02 & 10.08 & 10.58 \\
\hline 0.0800 & 2383. & -3.527 & 0.102 & 5.323 & 18.67 & 15.96 & 13.61 & 11.26 & 10.89 & 10.63 & 9.84 & 10.27 \\
\hline 0.0900 & 2627. & -3.268 & 0.113 & 5.285 & 16.98 & 14.86 & 12.67 & 10.72 & 10.30 & 10.03 & 9.40 & 9.72 \\
\hline 0.1000 & 2784. & -3.083 & 0.125 & 5.246 & 15.86 & 14.09 & 12.09 & 10.33 & 9.89 & 9.62 & 9.07 & 9.31 \\
\hline
\end{tabular}

Table 4. Same as Table 1 for 5 Gyr.

\begin{tabular}{|c|c|c|c|c|c|c|c|c|c|c|c|c|}
\hline$m / M_{\odot}$ & $T_{\text {eff }}$ & $\log L / L_{\odot}$ & $R / R_{\odot}$ & $\log g$ & $M_{V}$ & $M_{R}$ & $M_{I}$ & $M_{J}$ & $M_{H}$ & $M_{K}$ & $M_{L^{\prime}}$ & $M_{M}$ \\
\hline 0.0020 & 129. & -8.570 & 0.105 & 3.698 & 60.05 & 54.63 & 49.68 & 38.16 & 34.52 & 53.62 & 23.33 & 20.80 \\
\hline 0.0030 & 162. & -8.166 & 0.105 & 3.868 & 55.32 & 50.15 & 45.63 & 34.96 & 32.46 & 49.21 & 22.22 & 19.81 \\
\hline 0.0040 & 193. & -7.867 & 0.105 & 3.994 & 50.68 & 45.80 & 41.76 & 32.14 & 30.52 & 45.15 & 21.28 & 18.92 \\
\hline 0.0050 & 220. & -7.644 & 0.105 & 4.095 & 46.50 & 41.92 & 38.30 & 29.83 & 28.84 & 41.71 & 20.48 & 18.16 \\
\hline 0.0060 & 244. & -7.469 & 0.104 & 4.180 & 42.71 & 38.43 & 35.18 & 27.80 & 27.34 & 38.67 & 19.78 & 17.49 \\
\hline 0.0070 & 265. & -7.328 & 0.103 & 4.254 & 39.29 & 35.29 & 32.37 & 26.02 & 26.00 & 35.96 & 19.16 & 16.88 \\
\hline 0.0080 & 284. & -7.217 & 0.103 & 4.318 & 36.31 & 32.57 & 29.93 & 24.50 & 24.85 & 33.65 & 18.64 & 16.36 \\
\hline 0.0090 & 301. & -7.124 & 0.102 & 4.376 & 33.73 & 30.22 & 27.82 & 23.21 & 23.85 & 31.64 & 18.18 & 15.91 \\
\hline 0.0100 & 322. & -7.015 & 0.101 & 4.429 & 33.05 & 29.47 & 27.06 & 22.74 & 23.30 & 30.49 & 17.91 & 15.71 \\
\hline 0.0120 & 361. & -6.823 & 0.100 & 4.519 & 31.58 & 27.86 & 25.46 & 21.77 & 22.17 & 28.16 & 17.41 & 15.32 \\
\hline 0.0150 & 399. & -6.671 & 0.098 & 4.634 & 30.20 & 26.35 & 24.00 & 20.85 & 21.14 & 26.01 & 16.96 & 14.97 \\
\hline 0.0200 & 473. & -6.401 & 0.095 & 4.786 & 29.24 & 25.17 & 22.66 & 19.89 & 19.92 & 23.31 & 16.18 & 14.45 \\
\hline 0.0300 & 610. & -6.008 & 0.090 & 5.011 & 28.17 & 23.83 & 21.17 & 18.34 & 18.31 & 20.04 & 15.04 & 13.71 \\
\hline 0.0400 & 760. & -5.670 & 0.085 & 5.179 & 27.58 & 23.15 & 20.40 & 17.04 & 17.11 & 17.99 & 14.17 & 13.13 \\
\hline 0.0500 & 931. & -5.353 & 0.082 & 5.313 & 27.09 & 22.63 & 19.82 & 15.94 & 16.05 & 16.38 & 13.38 & 12.63 \\
\hline 0.0600 & 1120. & -5.058 & 0.079 & 5.418 & 26.44 & 22.03 & 19.20 & 15.01 & 15.13 & 15.15 & 12.73 & 12.27 \\
\hline 0.0700 & 1524. & -4.504 & 0.081 & 5.466 & 24.33 & 20.36 & 17.60 & 13.52 & 13.50 & 13.44 & 11.60 & 11.77 \\
\hline 0.0720 & 1712. & -4.278 & 0.083 & 5.453 & 23.11 & 19.36 & 16.75 & 12.97 & 12.85 & 12.80 & 11.16 & 11.54 \\
\hline 0.0750 & 2006. & -3.942 & 0.089 & 5.411 & 21.03 & 17.80 & 15.34 & 12.20 & 11.92 & 11.78 & 10.54 & 11.06 \\
\hline 0.0800 & 2320 . & -3.603 & 0.099 & 5.353 & 19.11 & 16.28 & 13.89 & 11.44 & 11.07 & 10.82 & 9.97 & 10.43 \\
\hline 0.0900 & 2622. & -3.275 & 0.113 & 5.289 & 17.02 & 14.88 & 12.70 & 10.73 & 10.31 & 10.04 & 9.42 & 9.73 \\
\hline 0.1000 & 2785. & -3.083 & 0.125 & 5.247 & 15.85 & 14.09 & 12.09 & 10.33 & 9.89 & 9.62 & 9.07 & 9.31 \\
\hline
\end{tabular}

elements $(\mathrm{Na}, \mathrm{K})$ at such pressures may be responsible for this discrepancy. No theory, however, exists to date for an accurate description of broadening of atomic lines by collisions with $\mathrm{H}_{2}$ and He. Attempts to improve current treatments are under progress (Burrows \& Volobuyev 2002).
The correct trend of colors and spectral properties predicted by the present models at wavelength $>1 \mu \mathrm{m}$, where most of the flux is emitted for the concerned range of $T_{\text {eff }}$, comfort us however with their reliability to describe extremely cool objects. 
Table 5. Same as Table 1 for 10 Gyr.

\begin{tabular}{ccccccccccccc}
\hline \hline$m / M_{\odot}$ & $T_{\text {eff }}$ & $\log L / L_{\odot}$ & $R / R_{\odot}$ & $\log g$ & $M_{V}$ & $M_{R}$ & $M_{I}$ & $M_{J}$ & $M_{H}$ & $M_{K}$ & $M_{L^{\prime}}$ & $M_{M}$ \\
\hline 0.0030 & 125. & -8.629 & 0.104 & 3.879 & 61.46 & 55.89 & 50.79 & 38.29 & 35.04 & 54.28 & 23.36 & 20.89 \\
0.0040 & 149. & -8.325 & 0.104 & 4.006 & 58.00 & 52.62 & 47.86 & 36.01 & 33.56 & 51.11 & 22.59 & 20.19 \\
0.0050 & 172. & -8.087 & 0.103 & 4.109 & 54.62 & 49.46 & 45.05 & 34.06 & 32.18 & 48.26 & 21.92 & 19.57 \\
0.0060 & 193. & -7.888 & 0.102 & 4.195 & 51.29 & 46.38 & 42.30 & 32.23 & 30.85 & 45.53 & 21.29 & 18.97 \\
0.0070 & 213. & -7.724 & 0.102 & 4.270 & 48.17 & 43.50 & 39.72 & 30.55 & 29.62 & 43.01 & 20.71 & 18.41 \\
0.0080 & 232. & -7.584 & 0.101 & 4.335 & 45.19 & 40.77 & 37.28 & 29.00 & 28.46 & 40.66 & 20.18 & 17.89 \\
0.0090 & 249. & -7.469 & 0.100 & 4.393 & 42.49 & 38.30 & 35.07 & 27.62 & 27.41 & 38.55 & 19.70 & 17.42 \\
0.0100 & 265. & -7.368 & 0.099 & 4.445 & 39.95 & 35.99 & 33.00 & 26.34 & 26.44 & 36.59 & 19.26 & 16.98 \\
0.0120 & 293. & -7.204 & 0.098 & 4.536 & 35.23 & 31.68 & 29.12 & 24.04 & 24.64 & 33.06 & 18.48 & 16.20 \\
0.0150 & 330. & -7.016 & 0.096 & 4.650 & 32.81 & 29.25 & 26.80 & 22.70 & 23.33 & 30.46 & 17.93 & 15.76 \\
0.0200 & 389. & -6.759 & 0.093 & 4.802 & 30.75 & 26.99 & 24.64 & 21.28 & 21.73 & 27.02 & 17.21 & 15.19 \\
0.0300 & 504. & -6.358 & 0.088 & 5.029 & 28.98 & 24.87 & 22.35 & 19.72 & 19.68 & 22.59 & 16.04 & 14.37 \\
0.0400 & 634. & -6.004 & 0.083 & 5.200 & 28.18 & 23.85 & 21.19 & 18.33 & 18.33 & 19.99 & 15.03 & 13.75 \\
0.0500 & 776. & -5.695 & 0.079 & 5.338 & 27.64 & 23.21 & 20.47 & 17.10 & 17.18 & 18.07 & 14.23 & 13.21 \\
0.0600 & 941. & -5.393 & 0.076 & 5.450 & 27.20 & 22.74 & 19.93 & 16.04 & 16.18 & 16.55 & 13.50 & 12.73 \\
0.0700 & 1289. & -4.832 & 0.078 & 5.503 & 25.69 & 21.45 & 18.60 & 14.37 & 14.43 & 14.36 & 12.27 & 12.08 \\
0.0720 & 1556. & -4.472 & 0.081 & 5.481 & 24.17 & 20.22 & 17.48 & 13.44 & 13.41 & 13.36 & 11.55 & 11.75 \\
0.0750 & 1997. & -3.954 & 0.089 & 5.415 & 21.10 & 17.85 & 15.39 & 12.23 & 11.95 & 11.81 & 10.56 & 11.08 \\
0.0800 & 2322. & -3.602 & 0.099 & 5.353 & 19.10 & 16.27 & 13.89 & 11.43 & 11.06 & 10.82 & 9.97 & 10.43 \\
0.0900 & 2624. & -3.274 & 0.113 & 5.289 & 17.01 & 14.88 & 12.69 & 10.73 & 10.31 & 10.04 & 9.41 & 9.73 \\
0.1000 & 2786. & -3.082 & 0.125 & 5.246 & 15.85 & 14.09 & 12.08 & 10.32 & 9.88 & 9.62 & 9.07 & 9.30 \\
\hline
\end{tabular}

\section{Irradiated models}

\subsection{Effect on atmosphere structure}

As mentioned in the introduction, a non-negligible fraction of EGPs orbit close to their parent star and their thermal and mechanical structure is affected by irradiation effects. Therefore, a general theory of cool substellar objects must take these effects into account. Recently Barman et al. (2001) have modeled irradiated atmospheres by including the impinging radiation field in the solution of the radiative transfer equation. As shown by these authors, for a given intrinsic luminosity, non-irradiated planets have very different temperature structures than irradiated planets. Thus, substituting non-irradiated atmospheric structures with $T_{\text {eff }}=T_{\text {eq }}$ (see definition below, Eq. (8)) for irradiated structures, as done up to now in the literature, yields incorrect inner boundary conditions for evolutionary calculations (see e.g. Fig. 13 of Barman et al. 2001). Given the present lack of an accurate treatment of atmospheric dust diffusion, the calculations were performed only for the DUSTY and COND limit cases, respectively. The results emphasize the strong dependence of the emergent spectrum and atmospheric structure on the presence or absence of dust. In the absence of dust, the impinging flux can penetrate in deeper layers of the planet atmosphere, affecting more drastically the inner structure of the planet than in the dusty case.

Except for a possible detection of sodium absorption in the atmosphere of HD 209458b (Charbonneau et al. 2002), no constraints on the atmospheric composition of EGPs are available at the present time. The only strong observational constraint available for irradiated models is the transit planet HD 209458b. The determination of its mass and radius provides a stringent test to irradiated atmosphere calculations and to the resulting structure and evolution. We thus apply our calculations of irradiated EGPs to HD 209458-like systems.

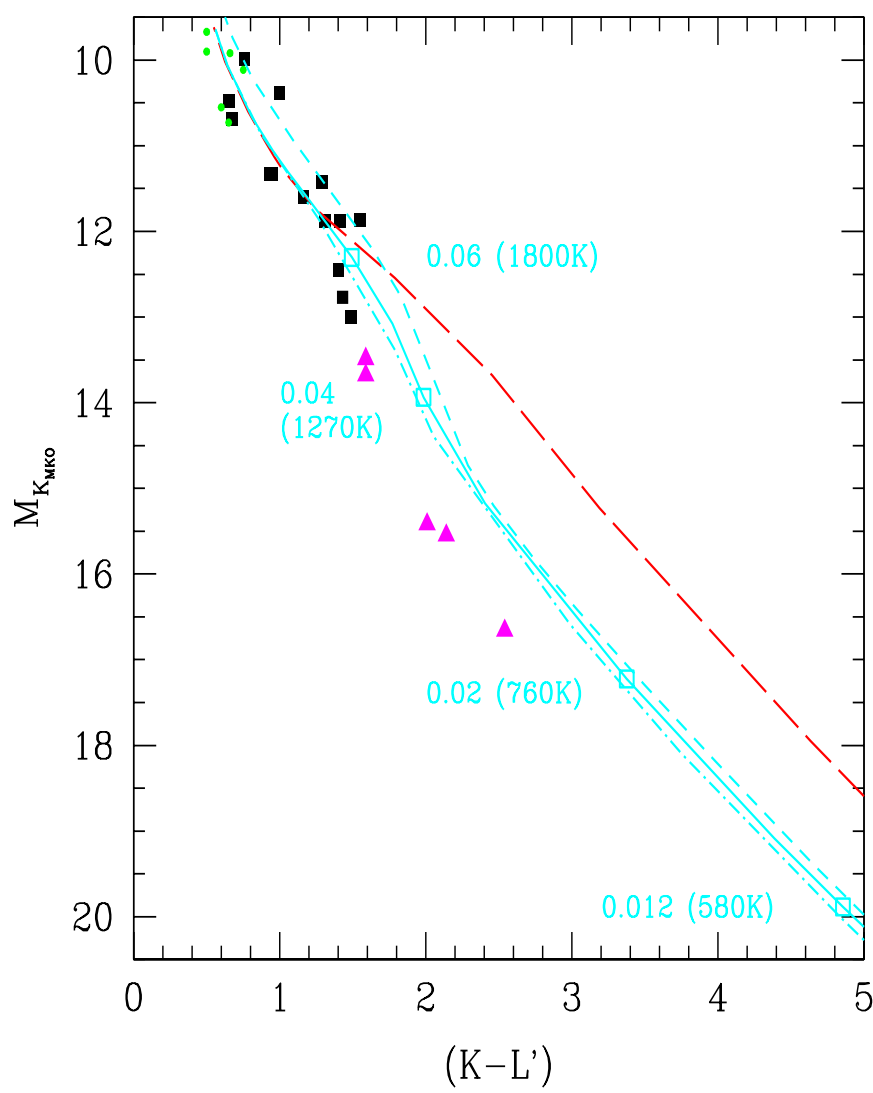

Fig. 3. Color - Magnitude diagram $\left(K-L^{\prime}\right)-M_{\mathrm{K}}$. $\mathrm{K}$ is in the MKO-NIR system. Observations are from Leggett et al. (2002a). Symbols are the same as in Fig. 2. A DUSTY isochrone of $1 \mathrm{Gyr}$ (Chabrier et al. 2000b) is indicated by the long-dashed line. The COND isochrones are displayed for $0.1 \mathrm{Gyr}$ (dash), $1 \mathrm{Gyr}$ (solid) and $10 \mathrm{Gyr}$ (dash-dot). Some masses (in $M_{\odot}$ ) and their corresponding $T_{\text {eff }}$ are indicated on the 1 Gyr COND isochrone by open squares. 


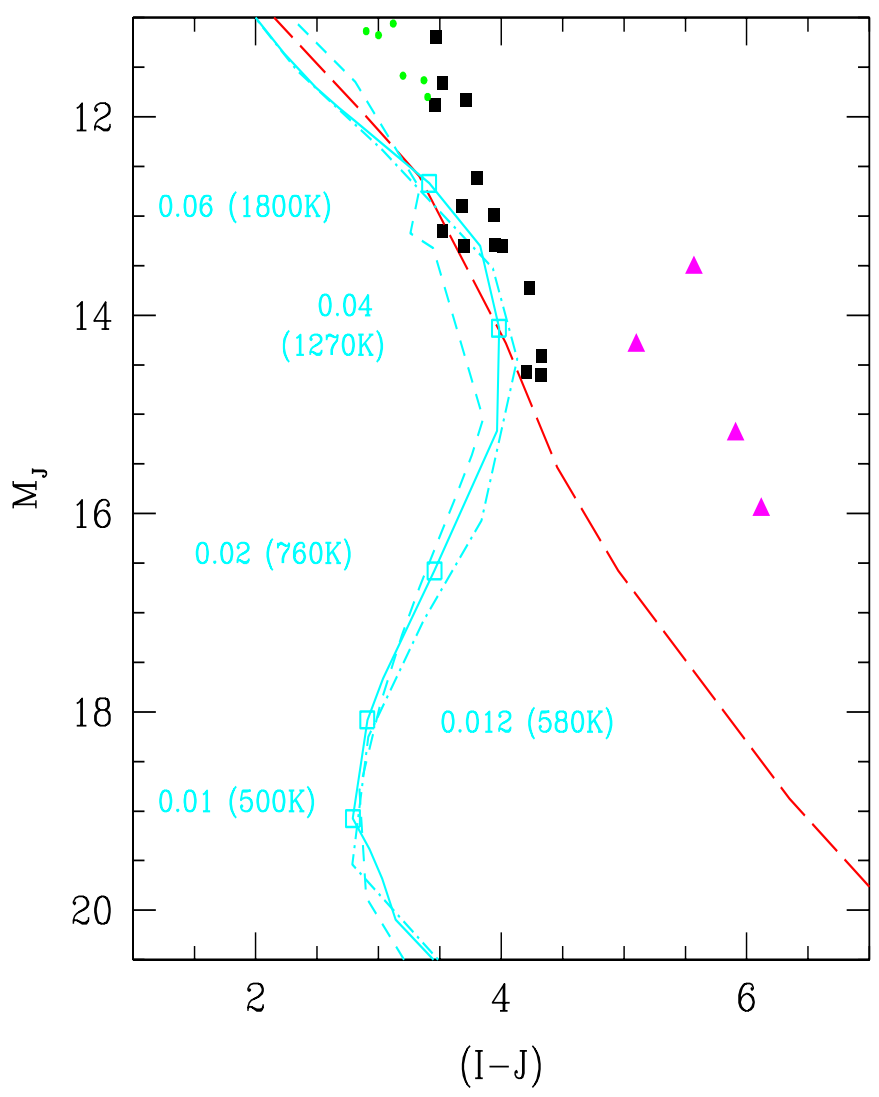

Fig. 4. Color - Magnitude diagram $(I-J)-M_{\mathrm{J}}$. Observations are from Leggett (1992) and Dahn et al. (2002). Symbols and curves are the same as in Fig. 3.

We have computed a grid of irradiated atmosphere models based on the COND input physics described in Sect. 2, as in Barman et al. (2001). Although more appropriate for EGPs with $T_{\text {eff }} \lesssim 1300 \mathrm{~K}$, the COND models maximise the effect of irradiation on the inner atmosphere structure and thus on the evolution of EPGs (Barman et al. 2001). The grid covers a wide range of $T_{\text {eff }}$ from $40 \mathrm{~K}$ to $100 \mathrm{~K}$, in steps of $20 \mathrm{~K}$, and from $100 \mathrm{~K}$ to $2800 \mathrm{~K}$, in steps of $100 \mathrm{~K}$. It covers a range of surface gravities from $\log g=2.5$ to $\log g=4.5$, in steps of 0.5 dex. We adopt the characteristics of HD 209458, assuming for the primary an effective temperature $T_{\text {eff } \star}=6000 \mathrm{~K}$, a radius $R_{\star}=1.18 R_{\odot}$ (Mazeh et al. 2000; Cody \& Sasselov 2002) and an orbital separation $a=0.046$ AU (Charbonneau et al. 2000). As in Barman et al. (2001), we make the simplifying assumptions that the impinging radiation field is isotropic and the incident flux $F_{\text {inc }}$ is redistributed only over the dayside, i.e.

$F_{\text {inc }}=\frac{1}{2}\left(\frac{R_{\star}}{a}\right)^{2} F_{\star}$,

where $F_{\star}$ is the total flux from the primary (see discussion in Sect. 4).

Before proceeding any further, we briefly re-specify definitions of fluxes (see e.g. Brett \& Smith 1993), since use of various terminologies leads to confusion. In all cases, the integrated net flux $F_{\text {net }}$, obtained from the solution of the transfer equation, is the intensity integrated over both in-coming and out-going directions $\mu$ ( $\mu=\cos \theta$, where $\theta$ is the angle of incidence). Assuming there is no extra source or sink of energy (e.g. no horizontal energy transfer), energy conservation implies that all the incident energy coming in must go out. Therefore, in the case of irradiation, the in-coming flux from the parent star cancels out the extra out-going, absorbed and reradiated flux due to the heating of the upper layers of the planet atmosphere (see Fig. 5). The in-coming flux at the surface is $F_{\text {in }}=-F_{\text {inc }}$ and the out-going flux at the surface is $F_{\text {out }}=F_{\text {inc }}+\sigma T_{\text {eff }}^{4}$, where $\sigma T_{\text {eff }}^{4}$ defines the intrinsic, unperturbed flux $\sigma T_{\text {eff }}^{4}$ of the initial, non-irradiated atmosphere structure. Energy conservation thus implies:

$F_{\text {net }}=F_{\text {out }}+F_{\text {in }}=\sigma T_{\text {eff }}^{4}$.

The non-irradiation case $\left(F_{\text {inc }}=0\right)$ corresponds to the usual condition $F_{\text {net }}=F_{\text {out }}=\sigma T_{\text {eff }}^{4}$.

Our atmosphere models, irradiated or not, are thus characterized by the parameters $T_{\text {eff }}$ and $g$. Of course, the same net flux $F_{\text {net }}$ corresponds to two different atmospheric structures, in the non-irradiated and irradiated case, because of the extra energy source $F_{\text {in }} \neq 0$ in the latter case (see Fig. 5). Given the above definitions, the net flux characterizes the intrinsic luminosity, i.e. the rate of energy released by the planet as it contracts and cools down:

$L_{\mathrm{int}}=4 \pi R_{\mathrm{p}}^{2} \sigma T_{\mathrm{eff}}^{4}=\int-T \frac{\mathrm{d} S}{\mathrm{~d} t} \mathrm{~d} m$.

This quantity determines the cooling properties of the planet for a given set of outer boundary conditions provided by the atmospheric profile (see Sect. 3.2 below). We stress that, in the case of irradiation, $T_{\text {eff }}$ does not characterize the total flux emitted by the planet, which is given by:

$F_{\text {out }}=\sigma T_{\text {eff }}^{4}+F_{\text {inc }}=\sigma T_{\text {eff }}^{4}+\frac{1}{2}\left(\frac{R_{\star}}{a}\right)^{2} F_{\star}$.

Note that $F_{\text {out }}$ is the important quantity for observers, since it characterises the total radiation of the planet, including both thermal and reflected parts of the flux. However, we do not focus on this quantity, since a forthcoming paper will be devoted to spectral properties of irradiated planets (Barman et al. 2003, in preparation).

For the sake of comparison with non-irradiated atmosphere profiles (see Barman et al. 2001), we also define the quantity $T_{\text {therm }}$ which characterises the thermal flux $\sigma T_{\text {therm }}^{4}$ emitted by the irradiated fraction of the planet (in the present case, the day side only). This quantity reads:

$\sigma T_{\text {therm }}^{4}=\sigma T_{\text {eff }}^{4}+(1-A) F_{\text {inc }}$,

where $A$ is the Bond albedo. According to the definitions above:

$F_{\text {out }}=\sigma T_{\text {therm }}^{4}+F_{\text {refl }}$,

where

$F_{\text {refl }}=A F_{\text {inc }}$

is the reflected part of the incident flux.

Within the conditions of the present calculations (Eq. (1), $a=0.046, T_{\text {eff } \star}=6000 \mathrm{~K}, R_{\star}=1.18 R_{\odot}$ ), our Bond albedo 
is close to 0.1 for the coolest models $\left(T_{\mathrm{eff}} \sim 100 \mathrm{~K}\right)$. A final quantity, often used in the literature, is the equilibrium temperature, $T_{\mathrm{eq}}$, which characterizes the planet's luminosity after having exhausted all its internal heat content (see e.g. Guillot et al. 1996; Saumon et al. 1996):

$$
\begin{aligned}
T_{\text {eq }}^{4}= & \frac{(1-A)}{\sigma} F_{\text {inc }}=\frac{(1-A)}{2 \sigma}\left(\frac{R_{\star}}{a}\right)^{2} F_{\star} \\
& \rightarrow T_{\text {therm }}^{4} \text { when } T_{\text {eff }} \rightarrow 0 .
\end{aligned}
$$

Note that given our definition of $F_{\text {inc }}, T_{\text {eq }}^{4}$ defined by Eq. (8) differs by a factor 2 from the definition usually used in the literature, because of the redistribution only over the day side. Note also that $T_{\text {eq }}$ and $T_{\text {therm }}$ differ significantly at young ages, when the intrinsic flux of the planet is not negligible.

The effect of irradiation on atmosphere structures is illustrated in Fig. 5 for different values of the effective temperature $T_{\text {eff }}$. As already stressed in Barman et al. (2001), an irradiated structure characterised by $T_{\text {therm }}$ can differ significantly from a non-irradiated structure at the same effective temperature $T_{\text {eff }}=T_{\text {therm }}$. This point (see also Seager \& Sasselov 1998; Guillot \& Showman 2002) emphasizes the fact that adopting outer boundary conditions, for evolutionary calculations, from atmospheric profiles of nonirradiated models with $T_{\text {eff }}=T_{\text {therm }}$, or $T_{\text {eff }}=T_{\text {eq }}$ (as e.g. Burrows et al. 2000), is incorrect and yields erroneous evolutionary properties for irradiated objects.

\subsection{Effect on evolution}

The main effect of irradiation on convective atmospheres and its consequences on evolution is well known (see Hubbard 1977; Brett \& Smith 1993; Guillot et al. 1996; Hubbard et al. 2002). The heating of the outer layers by the incident flux reduces the temperature gradient between these layers and the interior. They become radiative and the top of the convective zone is displaced to larger depths compared to the non-irradiated case, as clearly illustrated in Fig. 5. The inner atmosphere structure is hotter at a given pressure than the nonirradiated atmosphere model of same $T_{\text {eff }}$ (see Fig. 5). In order to match the same inner entropy, or the same values of $P$ and $T$, characteristic of the boundary layer between the interior structure and the irradiated atmosphere structure, characterized by a given $T_{\text {eff }}$ and $\log g$, one would need a nonirradiated atmosphere model with higher $T_{\text {eff }}$, i.e. a larger heat loss. Therefore, for a given planet heat content, i.e. internal entropy, the heat loss is reduced in the case of irradiation and the planet maintains a higher entropy for a longer time. Since for a given mass, the interior $(P, T)$ profile and thus the entropy fix the radius, the irradiated planet has a larger radius than the nonirradiated counterpart at a given time, starting from the same initial configuration. In other terms, gravitational contraction, which is the dominant source of energy of the planet, proceeds more slowly with irradiation than without it.

Our calculations proceed as for our low-mass star or brown dwarf calculations, by coupling the interior and atmosphere profile at a deep enough optical depth, which defines unequivocally the fundamental properties of the object, $m, R, T_{\mathrm{eff}}, L$ along its evolution $t$ (Chabrier \& Baraffe 1997). The boundary condition between inner and atmosphere structure is fixed

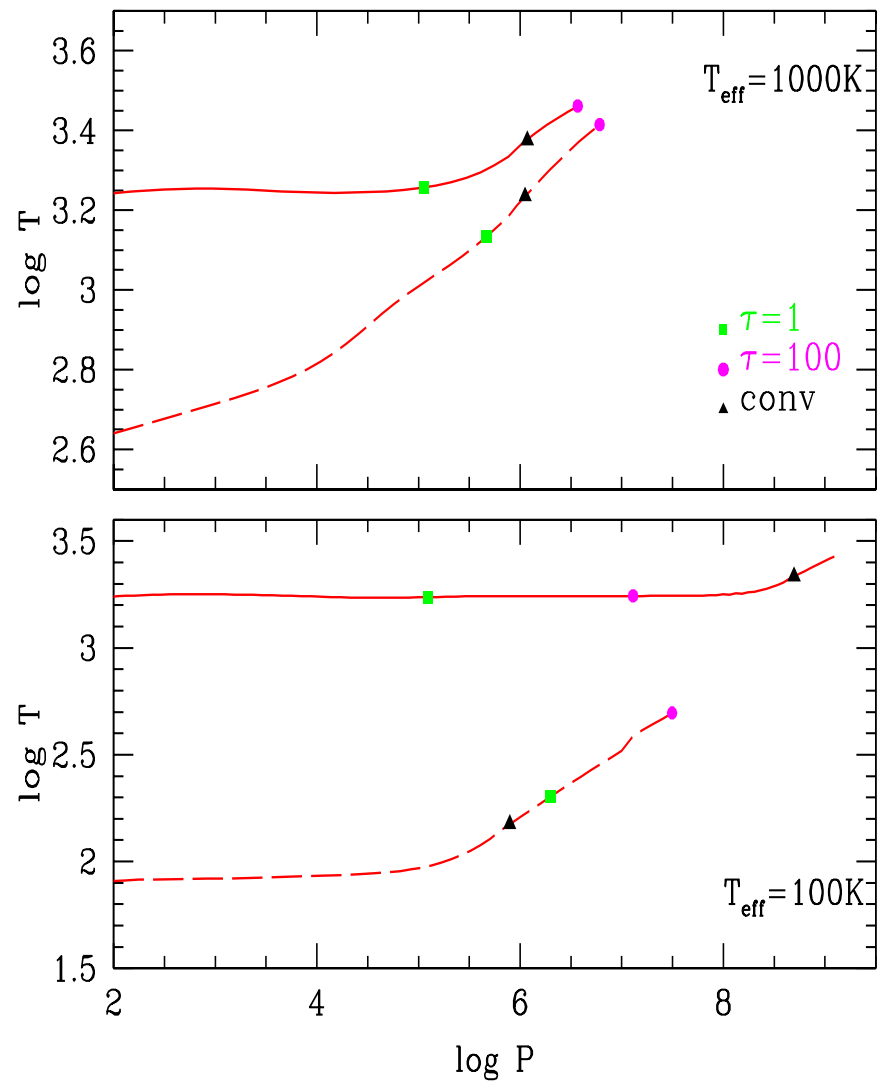

Fig. 5. Effect of irradiation on atmosphere profiles, $T(\mathrm{~K})$ versus $P\left(\mathrm{dyn} / \mathrm{cm}^{2}\right)$, characterized by a surface gravity $\log g=3.0, T_{\mathrm{eff}}=$ $1000 \mathrm{~K}$ (upper panel) and $T_{\text {eff }}=100 \mathrm{~K}$ (lower panel). Dashed lines correspond to nonirradiated structures. Solid lines are irradiated models at a separation $a=0.046 \mathrm{AU}$ from a primary with $T_{\text {eff }_{\star}}=6000 \mathrm{~K}$. The corresponding equilibrium temperature is $T_{\mathrm{eq}} \sim 1630 \mathrm{~K}$. The squares on the curve refer to optical depth, defined at $\lambda=1.2 \mu \mathrm{m}, \tau_{\mathrm{std}}=1$ and the circles to $\tau_{\text {std }}=100$. The triangles indicate the top of the convective zone.

at $\tau_{\text {std }}=100$, which corresponds to a range of pressure $P=$ $0.1-200$ bar for the whole range of atmosphere models ${ }^{1}$. The irradiated atmosphere models are integrated down to an optical depth $\tau_{\text {std }}=100$ for $T_{\text {eff }} \geq 1000 \mathrm{~K}$ and $\tau_{\text {std }}=10^{5}$ for $T_{\text {eff }}<$ $1000 \mathrm{~K}$. In both cases, this is deep enough to reach the top of the convective zone and to provide a good spatial resolution of these layers, even for the coolest models (see Fig. 5). In any case, the incident flux $F_{\text {inc }}$ drops to zero at $\tau_{\text {std }} \ll 50$, well above the deepest layers of the atmosphere models. Note that for the coolest atmospheric structures, convection does not reach the layers corresponding to $\tau_{\text {std }}=100$ (see Fig. 5). In that case, the radiative gradient in the interior is calculated with the Rosseland means of the same atmospheric opacities, for a consistent treatment between the interior and atmosphere thermal structures.

We have calculated the evolution of planets covering a range of mass from $0.5 M_{\mathrm{J}}$ to $10 M_{\mathrm{J}}$ with and without irradiation. The evolution of the radius as a function of time is shown in Fig. 6 for irradiated and nonirradiated EGPs of $1 M_{\mathrm{J}}$ and $10 M_{\mathrm{J}}$. As expected, the less massive the planet, the larger

\footnotetext{
${ }^{1} \tau_{\text {std }}$ is defined at $\lambda=1.2 \mu \mathrm{m}$.
} 


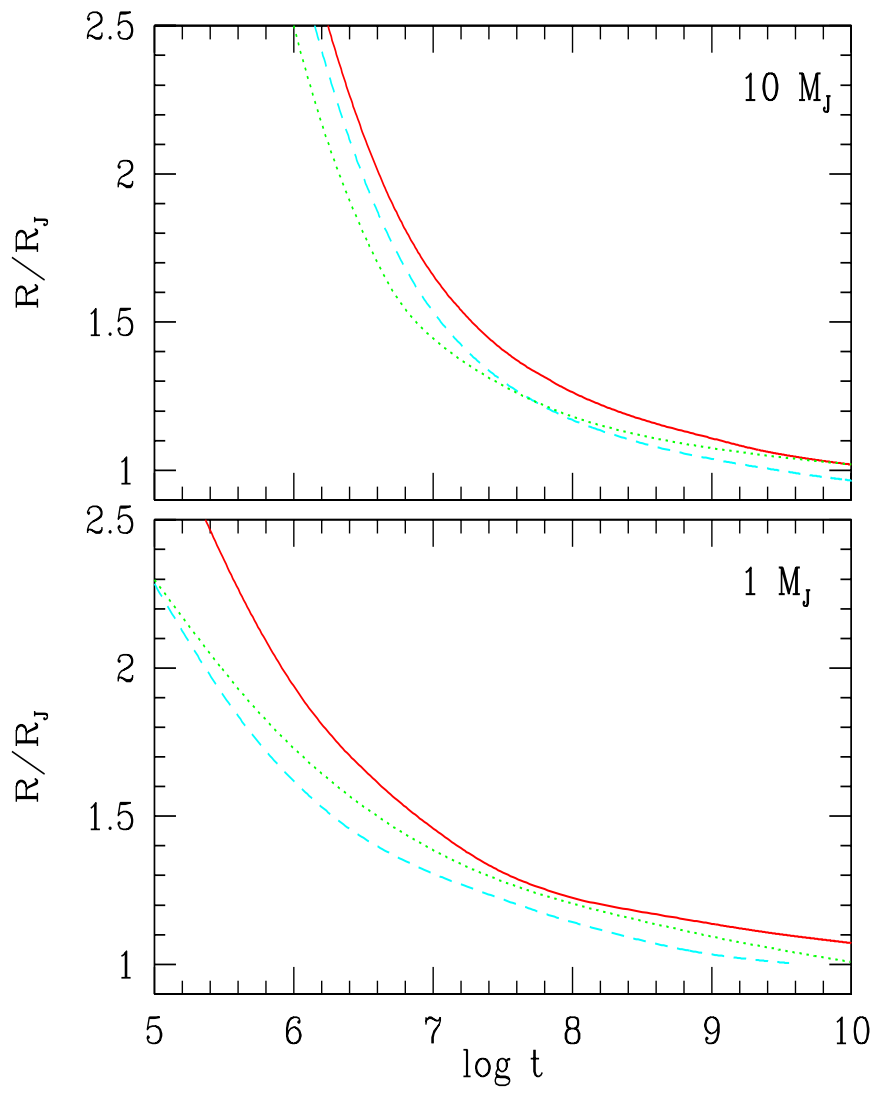

Fig. 6. Radius (in $R_{\mathrm{J}}$ ) versus age (in yr) for EGP masses of $1 M_{\mathrm{J}}$ (lower panel) and $10 M_{\mathrm{J}}$ (upper panel). The dashed lines are nonirradiated models. The solid lines are irradiated models at a separation $a=0.046 \mathrm{AU}$ from the parent star with $T_{\mathrm{eff}_{\star}}=6000 \mathrm{~K}$. The dotted lines are nonirradiated models from Burrows et al. (1997).

the effect of irradiation, for a given incident flux. At $1 \mathrm{Gyr}$, the $0.5 M_{\mathrm{J}}$ EGP has a $14 \%$ larger radius than its nonirradiated counterpart, whereas for the $1 M_{\mathrm{J}}$ (resp. $10 M_{\mathrm{J}}$ ), $R$ is only $10 \%$ larger (resp. 7\%). We also compare our COND models (the nonirradiated models) to the Burrows et al. (1997, hereafter B97) nonirradiated models. Significant differences appear at young ages $(<1 \mathrm{Gyr})$, due certainly to different initial conditions (see Baraffe et al. 2002). For ages $>1 \mathrm{Gyr}$ and $m \gtrsim 5 M_{\mathrm{J}}$, the differences between the B97 models and ours are of the order of the irradiation effects. This reflects the different input physics, mainly in the dust treatment and molecular opacities and illustrates the present uncertainties in the models. For $m \lesssim 5 M_{\mathrm{J}}$, however, irradiation effects become larger than the differences between the B97 and our models.

The specific case of HD 209458b, with a mass $m_{\mathrm{p}}=$ $0.69 M_{\mathrm{J}}$, is illustrated in Fig. 7. The intrinsic luminosity and corresponding effective temperature in the irradiated case (solid lines) are compared to the non-irradiated case (dashed lines). Starting from the same initial configuration in both cases, the heat loss is reduced at early ages in the case of irradiation, as expected. Consequently, the irradiated model evolves at larger entropy and radius than its non-irradiated counterpart. During the first Myr of evolution, both evolutionary sequences contract with increasing central density and temperature, the non-irradiated model being denser. The latter becomes partially
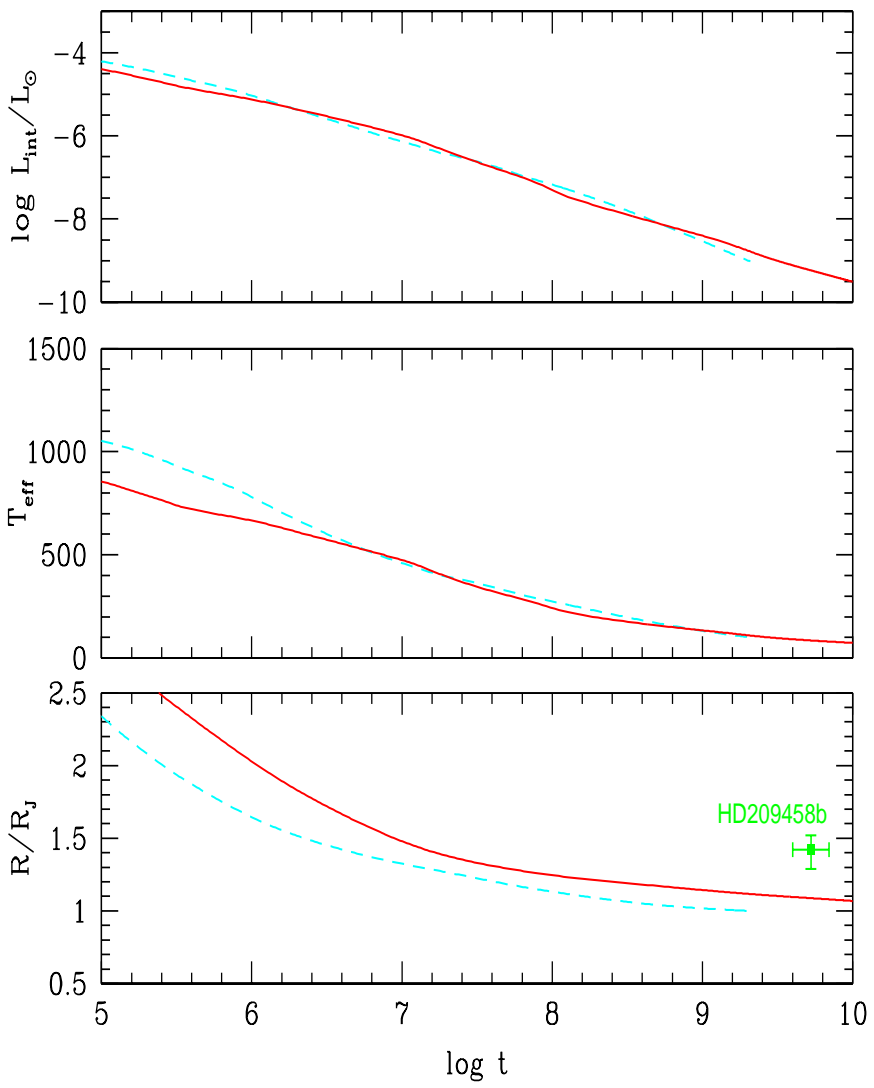

Fig. 7. Effect of irradiation on the evolution of a planet with $m_{\mathrm{p}}=$ $0.69 M_{\mathrm{J}}$ at a separation $a=0.046 \mathrm{AU}$ from its parent star with $T_{\text {eff }}=$ $6000 \mathrm{~K}$. The panels from top to bottom display respectively the intrinsic luminosity $L_{\text {int }}$, the effective temperature (in $\mathrm{K}$ ) and the radius versus time (in yr). The solid curves correspond to the irradiated case and the dashed curves to the nonirradiated counterpart. We recall that in the case of irradiation, $T_{\text {eff }}$ and $L_{\text {int }}$ do not characterize the total flux emitted by the planet. The position of HD 209458b in the lower panel is from Cody \& Sasselov (2002).

degenerate earlier, its contraction slows down and its heat loss becomes smaller than in the irradiated case (at $\log t \sim 6.2 \mathrm{yr}$ ). The situation reverses at $\log t \sim 7.4 \mathrm{yr}$ when the effect of partial degeneracy becomes important in the irradiated sequence. The age of HD 209458 is about 4-7 Gyr, according to Cody \& Sasselov (2002). At $5 \mathrm{Gyr}$, the irradiated sequence displayed in Fig. 7 predicts a radius $R=1.09 R_{\mathrm{J}}, 26 \%$ smaller than the observed value. Without including irradiation effects, the radius is $>30 \%$ than the observationally determined one. Note that the nonirradiated sequence stops at $T_{\text {eff }}=100 \mathrm{~K}$, corresponding to an age of $\sim 2 \mathrm{Gyr}$ and a radius $R=1 R_{\mathrm{J}}$. In the following section, we analyse the possible reasons for such a discrepancy.

\section{Discussion}

\subsection{Uncertainties of irradiated atmosphere/evolutionary models}

The question rises whether uncertainties of current models can explain the mismatch of HD 209458b predicted versus observed radius, and whether irradiation effects can still provide the solution to the problem. We first note that our choice of 
parameters for the irradiated atmosphere calculations certainly overestimates the effects of irradiation (see Sect. 3). We assume redistribution of the incident flux over the day-side of the planet only, without taking into account varying angles of incidence of the impinging flux. As shown in Brett \& Smith (1993), the effect of irradiation on the atmosphere will decrease with increasing angles of incidence. Note that adopting the maximum case, i.e. no redistribution at all of $F_{\text {inc }}$, affects significantly the outer atmospheric profile, but only slightly the inner profile. We did not consider horizontal energy flows, which may exist in a real planet receiving a varying amount of incident flux over its surface. But our assumption of isotropic incident flux, with the maximum amount of flux allowed, should provide an upper limit for the expected effects of irradiation on the evolution of a planet.

Although present atmosphere models still have some shortcomings, due either to incomplete molecular opacities or to dust treatment (see Sect. 2.2), the inner profiles of the irradiated models must be changed and heated drastically to provide the effect required on evolutionary models to match the radius of HD 209458b. Figure 7 indeed shows that the contraction of the irradiated sequence proceeds too rapidly after the first Myr of evolution to maintain a radius close to the observed value. At an age $t_{1}=1 \mathrm{Myr}$, the model has a radius $R_{1}=2 R_{\mathrm{J}}$ and an intrinsic luminosity $\log L_{1} / L_{\odot}=-5.12$, corresponding to $T_{\text {eff }}=$ $670 \mathrm{~K}$. This corresponds to a characteristic Kelvin-Helmholtz timescale $\tau_{\mathrm{KH}}=G m_{\mathrm{p}}^{2} /\left(R_{1} L_{1}\right) \sim 10 \mathrm{Myr}$. In fact, the model has already reached a radius of $R_{10}=1.48 R_{\mathrm{J}}$, about the radius of HD 209458b, after only $10 \mathrm{Myr}$ (see Fig. 7). In order to slow down the planet contraction to reach the observed value at $\sim 5 \mathrm{Gyr}$, the inner profiles of the present irradiated atmosphere models, for a given $T_{\text {eff }}$, should be drastically modified. Such a major modification seems unlikely, for a fixed incident flux. Note that assuming a constant flux from the parent star during the whole planet evolution overestimates the total incident flux received by the planet over $\sim 5$ Gyr. For a parent star mass $\sim 1.06 M_{\odot}$ (Cody \& Sasselov 2002), most of the star evolution after the first $1 \mathrm{Myr}$ proceeds at a luminosity $L$ smaller than its value at $5 \mathrm{Gyr}$.

Finally, possible uncertainties due to abundance effects, such as non-solar metallicity and/or helium abundance are not expected to affect significantly the present results. To estimate such uncertainties, we have computed a grid of irradiated atmosphere models for an over-solar metallicity $[\mathrm{M} / \mathrm{H}]=+0.3$ and the corresponding evolutionary sequence for a mass $m_{\mathrm{p}}=$ $0.69 M_{\mathrm{J}}$. After a few Gyr evolution, the radius of the later sequence is essentially the same as for the solar metallicity case. Given the optimization of irradiation effects provided by our assumptions, and the huge effect required on the inner profile to reduce the mismatch between observed and predicted radii, we do not expect uncertainties in the present models to be the source of the discrepancy.

\subsection{Observed versus theoretical radius}

The definition of the radius in low mass stars, brown dwarfs or isolated giant planets is usually not a matter of confusion, given the negligible extension of their atmosphere compared to the total radius of the object. The picture could be different in the case of irradiated atmospheres, where extension effects due to the large heating of the upper layers may not be negligible (Seager \& Sasselov 2000; Hubbard et al. 2001).

As discussed in Baschek et al. (1991), the condition of compactness for a photosphere in hydrostatic equilibrium is $H_{\mathrm{P}} / r \ll 1$, whit $H_{\mathrm{P}}$ the pressure scale height and $r$ the radial distance to the center. This condition is known to be perfectly fulfilled in (non-irradiated) low mass objects (see Chabrier \& Baraffe 1997), where the extension of the photosphere is usually less than $1 \%$ the total size of the object. Thus the radius can unambiguously be defined, quoting Baschek et al. (1991), as the distance of the atmosphere to the object center. In all our previous work, we fix the boundary condition between atmosphere and inner structure at $\tau_{\text {std }}=100$, knowing that $R\left(\tau_{\text {std }}=100\right)$ is essentially the same as $R\left(\tau_{\text {std }} \sim 1\right)$ (see Chabrier \& Baraffe 1997). As already mentioned, we define $\tau_{\text {std }}$ at $1.2 \mu \mathrm{m}$, which corresponds to the peak of the flux emitted by cool (non-irradiated) objects. Usually, the region where $\tau_{\text {std }} \sim 1$ is close to the region where $\tau_{\text {Rosseland }} \sim 1$ (for the present irradiated models as well). Above this region, the atmosphere contains little mass and contributes negligibly to the luminosity. The evolutionary calculations for irradiated models presented in Sect. 3.2 determine also the radius at $\tau_{\text {std }}=100$. Within the present assumptions of irradiation, the atmospheric extension between $\tau_{\text {std }}=100$ and $\tau_{\text {std }} \sim 1$ represents only $1-2 \%$ of the total radius. The presently calculated theoretical radius is thus essentially equivalent to a photospheric radius at $1.2 \mu \mathrm{m}$.

However, the observed radius of HD 209458 b (e.g. the one estimated by Cody \& Sasselov 2002) is based on the analysis of optical light curves. It corresponds to a region of the atmosphere where the optical depth is near unity at $0.6 \mu \mathrm{m}$, which is near $\tau_{\text {std }}=10^{-2}$ in our atmosphere models. Therefore, the radius predicted by the evolutionary models is not equivalent to the observed radius. If the atmospheric extension and the opacity of the atmosphere are large at the observed wavelengths, the measured radius could be different from the radius predicted by the evolutionary calculation. However, based on our irradiated atmosphere models with the gravity predicted by the evolution and the age of HD $209458 \mathrm{~b}$ (i.e. $\log g=3.2$ ), the atmospheric extension between $\tau_{\text {std }}=100$ and $\tau_{\text {std }}=10^{-2}$ (where the optical depth is close to unity at $0.6 \mu \mathrm{m}$ ) is very small, namely $0.05 R_{\mathrm{J}}$, compared to the overall radius (Barman et al. 2003, in preparation). Adding this value to the radius predicted by the models at 5 Gyr yields an optical-depth radius at $0.6 \mu \mathrm{m}$ of $\sim 1.14 R_{\mathrm{J}}$, still $22 \%$ less than the observed value. For younger planets or planets undergoing stronger irradiation effects, with much lower gravities, the extension will be more important and should be taken into account for a consistent comparison between theoretical and observed radii, as already stressed by Seager \& Sasselov (2000) and Hubbard et al. (2001).

In the same vein, Hubbard et al. (2001) estimate a radius of $94430 \mathrm{~km}\left(1.32 R_{\mathrm{J}}\right)$ at a pressure of 1 bar, based on a detailed analysis of physical effects influencing the observed light curve of HD 209458b. At $5 \mathrm{Gyr}$, our models predict a radius at 1 bar of $\sim 1.1 R_{\mathrm{J}}, 18 \%$ smaller than the 


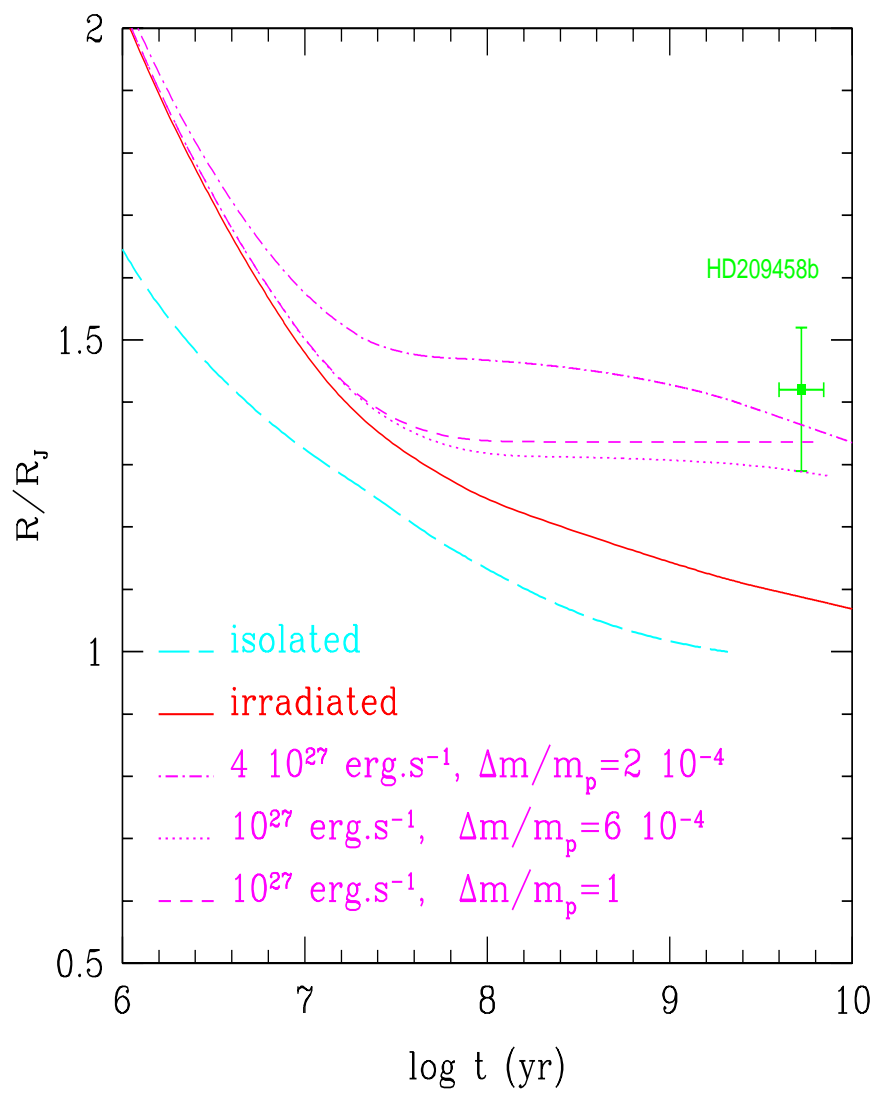

Fig. 8. Effect of extra source of energy dissipation on the evolution of a planet with mass $m_{\mathrm{p}}=0.69 M_{\mathrm{J}}$. The solid and long-dashed lines correspond to the irradiated and non-irradiated sequences respectively, with no extra source of energy dissipation. The other curves correspond to irradiated sequences with a total amount of additional energy $L_{\text {extra }}$ (in $\mathrm{erg} \mathrm{s}^{-1}$ ) deposited in layers between the surface and the mass shell $m_{\mathrm{p}}-\Delta m$, as indicated in figure (see text).

Hubbard et al. (2001) estimate. Such a discrepancy is consistent with the afore-mentioned mismatch for the radius at $0.6 \mu \mathrm{m}$.

\subsection{Other sources of energy deposit}

If irradiation effects alone do not explain the large observed radius of HD 209458b, other sources of energy must be invoked. Tidal interactions between the star and the planet can provide a source of energy associated to the synchronization and/or circularization of the planet orbit, dissipated within the planet (Lubow et al. 1997; Rieutord \& Zahn 1997; Bodenheimer et al. 2001). However, as discussed recently by Guillot \& Showman (2002) and Showman \& Guillot (2002), these processes are efficient only during the early stages of the planet evolution. Estimates based on the current understanding of such processes yield typical circularisation timescale $\tau_{\text {circ }} \sim 10^{8} \mathrm{yr}$ (Bodenheimer et al. 2001) and synchronisation timescale $\tau_{\text {syn }}<10^{8} \mathrm{yr}$ (Lubow et al. 1997; Rieutord \& Zahn 1997). Such an energy source seems thus unlikely to slow down the long term evolution of the planet, unless a second planet orbiting HD 209458a is present. Such a detection has been suggested very recently in the literature (Bodenheimer et al. 2003) but remains to be confirmed unambiguously.
Showman \& Guillot (2002) suggested that downward transport of kinetic energy produced by atmospheric circulation could be dissipated in the planet interior, leading to a substantial deposit of energy. Within the present input physics and treatment of irradiation, we can estimate the amount of energy required to reach the radius of HD 209458b. As in Guillot \& Showman (2002), we arbitrarily add an extra term of energy generation $\dot{\epsilon}_{\text {extra }}$ in the energy equation at different depths. We have explored several cases displayed in Fig. 8. We add a total amount $L_{\text {extra }}=\int \dot{\epsilon}_{\text {extra }} \mathrm{d} m$ in a region of mass $\Delta m$ enclosed between the surface and an arbitrary depth at mass shell $m_{1}$ (i.e. $\left.\Delta m=m_{\mathrm{p}}-m_{1}\right)$. Various tests indicate that an amount of energy $L_{\text {extra }} \sim 10^{27}-5 \times 10^{27} \mathrm{erg} \mathrm{s}^{-1}$ dissipated along the internal adiabat yields a radius within the error bars of the observed value (see Fig. 8). As expected, the larger the fraction of $L_{\text {extra }}$ deposited in the convective layers, the more important the effect. Note that the case displayed in Fig. 8 with $L_{\text {extra }} \sim 10^{27} \mathrm{erg} \mathrm{s}^{-1}$ dissipated all over the star $\left(\Delta m / m_{\mathrm{p}}=1\right.$, dashed curve $)$ is equivalent to depositing the same amount of energy only at the very center. Our quantitative estimates are in general agreement with Guillot \& Showman (2002). Such an amount of energy represents more than 100 times the intrinsic luminosity $L_{\text {int }}$ of the planet, which is $\sim 10^{25} \mathrm{erg} \mathrm{s}^{-1}$ at $1 \mathrm{Gyr}$ and $\sim 2 \times 10^{24} \mathrm{erg} \mathrm{s}^{-1}$ at $5 \mathrm{Gyr}$ (see Fig. 7). However, it represents only $\sim 1 \%$ of the incoming luminosity, $L_{\text {inc }}=2 \pi R_{\mathrm{p}}^{2} F_{\text {inc }} \sim 10^{29} \mathrm{erg} \mathrm{s}^{-1}$, which largely dominates the planet energetic balance. Thus, an alternative possibility is the release of an external source of energy caused by the incident radiation (see e.g. Showman \& Guillot 2002). As illustrated in Fig. 8, however, the extra source of energy must be dissipated at the top of the internal adiabat, i.e. at a much deeper level than the penetration of the incident photons $(\gg \tau=1)$.

\section{Conclusion}

We have presented calculations describing the evolution of cool brown dwarfs and extra-solar gaseous planets. The present models reproduce the main trends of observed methane-dwarfs in near-IR color-magnitude diagrams $(J-K, K-L)$. Problems still remain at wavelengths $<1 \mu \mathrm{m}$, with a flux excess predicted in the I-bandpass. The treatment of atomic line broadening in such dense objects may be the source of the present discrepancy. The models fail to reproduce the coolest L-dwarfs and a detailed treatment of dust diffusion/sedimentation is required for a correct description of the transition region between Land T- dwarfs. Work is in progress in this direction.

We have included the effects of irradiation, coupling irradiated atmosphere profile and inner structure, and providing consistent evolutionary models for irradiated planets. The effect of irradiation are shown to modify significantly the mechanical (mass-radius) and thermal evolution of irradiated EGPs. However, a significant discrepancy $(26 \%)$ remains between the theoretical and observed radii of the transit planet HD 209458b. We have explored possible uncertainties inherent in the models to explain such a discrepancy. Although solving these uncertainties may modify the outer structure of the models (extension of the atmosphere, albedo) and perhaps slightly reduce the discrepancy, none of the uncertainties is likely to modify 
significantly the inner entropy profile of the models, which determines the radius of the planet. Indeed, a drastic modification on the inner thermal structure is required to bring the theoretical radius in agreement with the observed one.

In summary, we do not expect irradiation effects alone to explain the large observed radius of HD 209458b. In the same vein, tidal interactions will affect only the early stages of evolution of the planet but will probably be dissipated too rapidly to affect the long term contraction of the object. Other sources of energy, representing about 100 times the intrinsic luminosity of the planet, seem to be required to explain the observed radius. The first extra-solar planet transit thus remains a challenge for theory. Detection of other transits is now crucial to conclude whether HD 209458 is a peculiar system, whether a second planetary companion is confirmed or not, or whether we are missing something in the current understanding of close-in giant planets.

Note: Isochrones for $t \geq 1 \mathrm{Myr}$ of the COND models (from $0.5 M_{\mathrm{J}}$ to $0.1 M_{\odot}$ ) are available at: http: //www . ens-lyon. fr/ ibaraffe/CONDQ3_models

Acknowledgements. We are very grateful to $\mathrm{H}$. Harris and S. Leggett for providing data under ascii files and to Doug Lin for mentioning the possible detection of a second planet. We thank our anonymous referee for valuable comments. I.B thanks the MaxPlanck-Institut für Astrophysik in Garching for hospitality during elaboration of part of this work. This research was supported in part by the LTSA grant NAG 5-3435, the NASA EPSCor grant to Wichita State University, NSF grants AST-9720704 and AST-0086246, NASA grants NAG5-8425, NAG5-9222, as well as NASA/JPL grant 961582 to the University of Georgia. This work was supported in part by the Pôle Scientifique de Modélisation Numérique at ENS-Lyon. Some of the calculations presented in this paper were performed on the IBM pSeries 690 of the Norddeutscher Verbund für Hoch- und Höchstleistungsrechnen (HLRN), on the IBM SP "Blue Horizon" of the San Diego Supercomputer Center (SDSC), with support from the National Science Foundation, on the IBM SP and the Cray T3E of the NERSC with support from the DoE, and using the computer facilities at Centre d'Études Nucléaires de Grenoble, CINES and IDRIS. We thank all these institutions for a generous allocation of computer time.

\section{References}

Allard, F., Hauschildt, P. H., Baraffe, I., \& Chabrier, G. 1996, ApJ, 465, L123

Allard, F., Hauschildt, P. H., Alexander, D. R., \& Starrfield, S. 1997, ARA\&A, 35, 137

Allard, F., Hauschildt, P. H., Alexander, D. R., Tamanai, A., \& Schweitzer, A. 2001, ApJ, 556, 357

Allard, F. 2002, IAU Symposium 211: Brown Dwarfs, ed. E. L. Martin, Publications of the Astronomical Society of the Pacific, Conf. Ser.

Ackerman, A. S., \& Marley, M. S. 2001, ApJ, 556, 872

Baraffe, I., Chabrier, G., Allard, F., \& Hauschildt, P. H. 2002, A\&A, 337,403

Baraffe, I., Chabrier, G., Allard, F., \& Hauschildt, P. H. 2002, A\&A, 382,563

Barman, T., Hauschildt, P. H., \& Allard, F. 2001, ApJ, 556, 885
Baschek, B., Scholz, M., \& Wehrse, R. 1991, A\&A, 246, 374

Bodenheimer, P., Lin, D. N. C., \& Mardling, R. A. 2001, ApJ, 548, 466

Bodenheimer, P., Laughlin, G., \& Lin, D. N. C. 2003, ApJ, submitted

Brett, J. M., \& Smith, R. C. 1993, MNRAS, 264, 641

Burrows, A., \& Volobuyev, M. 2002, ApJ, in press [astro-ph/0210086]

Burrows, A., Marley, M., Hubbard, W. B., et al. 1997, ApJ, 491, 856 (B97)

Burrows, A., Marley, M., \& Sharp, C. M. 2000, ApJ, 531, 438

Burrows, A., Guillot, T., Hubbard, W. B., et al. 2000, ApJ, 534, L97

Burgasser, A. J., Kirkpatrick, J. D., Brown, M. E., et al. 1999, ApJ, 422, L65

Burgasser, A. J., Marley, M., Ackerman, A. S., et al. 2002, ApJ, 571, L151

Chabrier, G., \& Baraffe, I. 1997, A\&A, 327, 1039

Chabrier, G., \& Baraffe, I. 2000, ARA\&A, 38, 337

Chabrier, G., Baraffe, I., Allard, F., \& Hauschildt, P. H. 2000a, ApJ, 542, L119

Chabrier, G., Baraffe, I., Allard, F., \& Hauschildt, P. H. 2000b, ApJ, 542,464

Charbonneau, D., Brown, T. M., Latham, D. W., \& Mayor, M. 2000, ApJ, 529, L45

Charbonneau, D., Brown, T. M., Noyes, R. W., \& Gilliland, R. L. 2002, ApJ, 568, 377

Cody, A. M., \& Sasselov, D. D. 2002, ApJ, 569, 451

Cuby, J. G., Saracco, P., Moorwood, A. F. M., et al. 1999, A\&A, 349, L41

Dahn, C. C., Harris, H. C., Vrba, F. J., et al. 2002, AJ, 124, 1170

Els, S. G., Sterzik, M. F., Marchis, F., et al. 2001, A\&A, 370, L1

Goldman, B., et al. 1999, A\&A, 351, L5

Guillot, T., Chabrier, G., Gautier, D., \& Morel, P. 1995, ApJ, 450, 463

Guillot, T., Burrows, A., Hubbard, W. B., Lunine, J. I., \& Saumon, D. 1996, ApJ, 459, L35

Guillot, T., \& Showman, A. P. 2002, A\&A, 385, 156

Hubbard, W. B. 1977, Icarus, 30, 305

Hubbard, W. B., Fortney, J. J., Lunine, J. I., et al. 2001, ApJ, 560, 413

Hubbard, W. B., Burrows, A., \& Lunine, J. I. 2002, ARA\&A, submitted

Leggett, S. K. 1992, ApJS, 82, 351

Leggett, S. K., Golimowski, X. F., Geballe, T. R., et al. 2002a, ApJ, 564,452

Leggett, S. K., Hauschildt, P. H., Allard, F., Geballe, T. R., \& Baron, E. 2002b, MNRAS, 332, 78L

Lubow, S. H., Tout, C. A., \& Livio, M. 1997, ApJ, 484, 866

Marley, M. S., Seager, S., Saumon, D., et al. 2002, ApJ, 568, 335

Mayor, M., \& Queloz, D. 1995, Nature, 378, 355

Mazeh, T., Naef, D., Torres, G., et al. 2000, ApJ, 532, L55

Oppenheimer, B. R., Kulkarni, S. R., Matthews, K., \& Nakajima, T. 1995, Science, 270, 1478

Rieutord, M., \& Zahn, J. P. 1997, ApJ, 474, 760

Saumon, D., Hubbard, W. B., Burrows, A., Guillot, T., Lunine, J. I., \& Chabrier, G. 1996, ApJ, 460, 993

Showman, A. P., \& Guillot, T. 2002, A\&A, 385, 166

Seager, S., \& Sasselov, D. D. 1998, ApJ, 502, L157

Seager, S., \& Sasselov, D. D. 2000, ApJ, 537, 916

Strauss, M. A., Fan, X., Gunn, J. E., et al. 1999, ApJ, 522, L61

Sudarsky, D., Burrows, A., \& Pinto, P. 2000, ApJ, 540, 504

Tsuji, T., Ohnaka, K., \& Aoki, W. 1999, A\&A, 520, L119

Zapatero Osorio, M. R., Béjar, V. J. S., Martín, E. L., et al. 2002, ApJ, 578,536 